\title{
détection des cavités souterraines par des méthodes géophysiques
}

\author{
par \\ Richard Lagabrielle \\ Ingénieur Civil des Mines \\ Assistant, Laboratoire Central des Ponts et Chaussées \\ et

\section{Marcel Rat} \\ Ingénieur des Ponts et Chaussées \\ Adjoint au Chef du Département Géotechnique \\ Laboratoire Central des Ponts et Chaussées
}

L'urbanisation croissante, le développement des moyens de communication, conduisent l'ingénieur à construire dans des zones contenant des cavités, soit naturelles (dissolution de gypse, calcaire), soit artificielles (carrière ayant été généralement exploitée pour fournir des matériaux de construction). Or, la détection des cavités souterraines est l'un des problèmes de la reconnaissance géologique les plus difficiles à résoudre.

La méthode la plus utilisée actuellement est la microgravimétrie, dont on connaît assez bien les limites. Dans certains cas particuliers, les méthodes sismiques réfraction ou électriques donnent des résultats positifs. Pour améliorer les moyens, la Délégation Générale à la Recherche Scientifique et Technique a financé un programme de recherche", sur de nouvelles méthodes, qui ont pu être comparées sur un site donné. Après avoir décrit ce site, pour chacune des méthodes expérimentées, nous donnerons le principe et les résultats obtenus.

\section{Description du site}

Le site est situé sur le plateau de Trappes, à proximité de Versailles, au lieu-dit "Troux». Les cavités que l'on a découvertes sont des "marnières", creusées par l'homme jusqu'au siècle dernier, le matériau exploité était les marnes du Calcaire de Beauce utilisées pour amender les terrains agricoles de surface, constitués par les limons des plateaux.

Les marnières ont un diamètre de l'ordre de $6 \mathrm{~m}$, elles étaient semble-t-il exploitées sans soutènement, ce qui explique leur taille réduite. Le toit était constitué par un banc plus induré de calcaire de 10 à $30 \mathrm{~cm}$ d'épaisseur (fig. 1). Si les chambres avaient été plus grandes, le toit

- ont participé à cette action :

le B.R.G.M. (Bureau de Recherches Géologiques et Minières) ;

- le L.G.A.O. (Laboratoire de Géophysique appliquée et d'Océanographie de l'Université de Bordeaux): Étude du trainé sismique :

- le L.C.P.C. (Laboratoire Central des Ponts et Chaussés): Étude de la télédiagraphie électrique :

- le Laboratoire de radiopropagation de I'Université de Lille : Étude d'une méthode électromagnétique par focalisation des courants ;

- le C.R.G. (Centre de Recherches Géophysiques): Étude de la M.T.A. aurait sans doute cédé. La hauteur exploitée est de l'ordre de 2 à $3 \mathrm{~m}$. Les chambres étaient isolées ou en groupe de 2 à 4 réparties en trèfle autour du puits d'accès. Les puits, creusés à la main, devaient avoir un diamètre de $1 \mathrm{~m}$ à $1,50 \mathrm{~m}$, ils ont été comblés et ont complètement disparu du paysage, leur faible emprise les rend presque impossibles à déceler (cependant nous en avons retrouvé un, après avoir d'abord découvert la marnière correspondante ; nous étions à ce moment là certains de sa présence dans un rayon de $10 \mathrm{~m}$ et le problème était très simplifié).

La profondeur des marnières est variable, elle dépend de la position des marnes du Calcaire de Beauce, et elle est limitée vers le bas par la nappe phréatique, l'exploitation se faisant toujours hors d'eau. Sur le site, on les trouve entre 10 et $15 \mathrm{~m}$ de profondeur.

Ces cavités sont donc petites: un calcul élémentaire permet en effet de montrer que l'anomalie gravimétrique

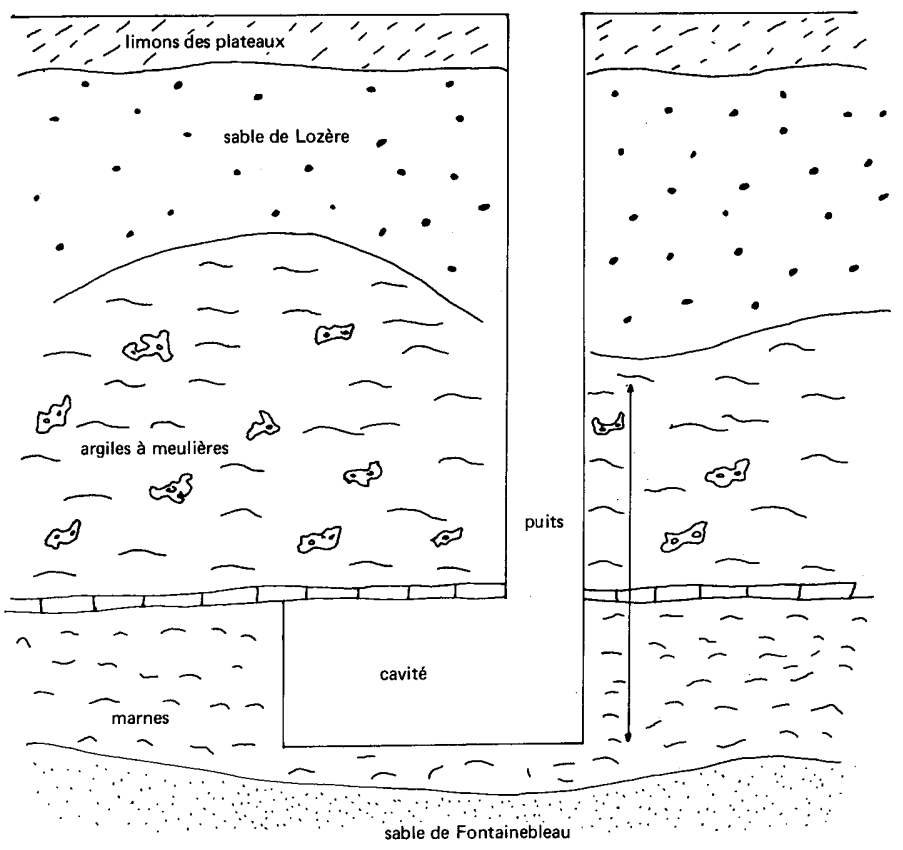

Fig. 1 Marnière avant le comblement du puits d'accès Coupe géologique schématique 
qu'elles créent est au maximum de 10 microgal, alors que la limite de cette méthode classique, et efficace dans d'autres conditions, est supérieure à 20 microgal. Elles sont cependant dangereuses pour les constructions, leur effondrement constituant des entonnoirs de plus de 10 mètres de diamètre.

\section{La tolédiagraphie électrique (T.D.E.)}

\subsection{Principe}

La méthode consiste à émettre une onde électromagnétique au moyen d'un dipôle électrique horizontal (D.E.H.) à la surface du sol et orienté vers l'axe du forage, matérialisé par deux électrodes par lesquelles on injecte un courant alternatif, d'amplitude constante. Simultanément une sonde portant deux électrodes permet la mesure de la composante verticale du champ électrique le long de la paroi du forage.

Si le terrain est électriquement homogène, la différence de potentiel entre les électrodes de la sonde est donnée par la formule suivante

$$
\Delta V=\frac{p}{2 \pi}\left(\frac{e^{-\frac{R 11}{\delta}}}{R 11}-\frac{e^{-\frac{R 21}{\delta}}}{R 21}-\frac{e^{-\frac{R 12}{\delta}}}{R 12}+\frac{e^{-\frac{R 22}{\delta}}}{R 22}\right)
$$

$R_{i j}=$ distance électrode $i$ de la sonde à l'électrode $j$ de l'émetteur.

(2) $\delta=500 \sqrt{\frac{\rho}{f}}$ est la profondeur de pénétration de l'onde.

$\rho:$ résistivité

$f$ : fréquence

Si le terrain est hétérogène, la formule (1) permet de définir une résistivité équivalente : c'est la résistivité du terrain homogène dans lequel, pour la même implantation des électrodes et le même courant injecté, on mesurerait la même différence de potentiel entre les électrodes de la sonde. Ce concept de résistivité équivalente est à la base de la technique d'interprétation des mesures, dont nous reparlerons plus bas.

\section{2 Étude sur modèle réduit}

Avant l'expérimentation sur le terrain, nous avons étudié la faisabilité du procédé sur un modèle réduit. La résistivité $\rho_{0}$ de l'électrolyte est connue, si $\rho_{1}$ est la résistivité équivalente mesurée, on peut définir un indice d'anomalie :

$$
\Delta=\frac{\rho_{0}-\rho_{1}}{\rho_{0}}
$$

Si l'on suppose que sur le terrain on saura mesurer un indice d'anomalie égal à $5 \%$, on peut tracer la courbe distance-profondeur de la cavité qui donne cet indice d'anomalie. Elle représente les limites de détectabilité d'une hétérogénéité isolante dans le sol. Sur la figure 2 , nous donnons les résultats obtenus pour des cylindres de $5 \mathrm{~cm}$ de diamètre. II suffit de multiplier par le même coefficient ${ }^{*}$ les distances $R, Z$ et le diamètre du cylindre pour obtenir la limite de la méthode.

\subsection{Principes d'interprétation}

L'équation (1) concernait un terrain homogène, l'étude sur cuve analogique servait à prévoir le résultat des mesures dans le cas d'une cavité isolée dans un milieu par ailleurs homogène. Ces modèles simples ont le mérite de permettre l'établissement d'une théorie, ils ne rendent que partiellement compte de la réalité. Nous avons en effet recherché des cavités situées dans des terrains stratifiés. II est possible de calculer théoriquement la variation du champ électrique vertical dans le cas d'un terrain stratifié horizontal, mais le calcul, par ailleurs lourd, nécessite la connaissance des épaisseurs et résistivités des différentes couches. Nous avons préféré procéder autrement en remarquant que dans un terrain stratifié horizontal, il existe une symétrie cylindrique autour du forage vertical. La présence d'une cavité rompt la symétrie. La recherche

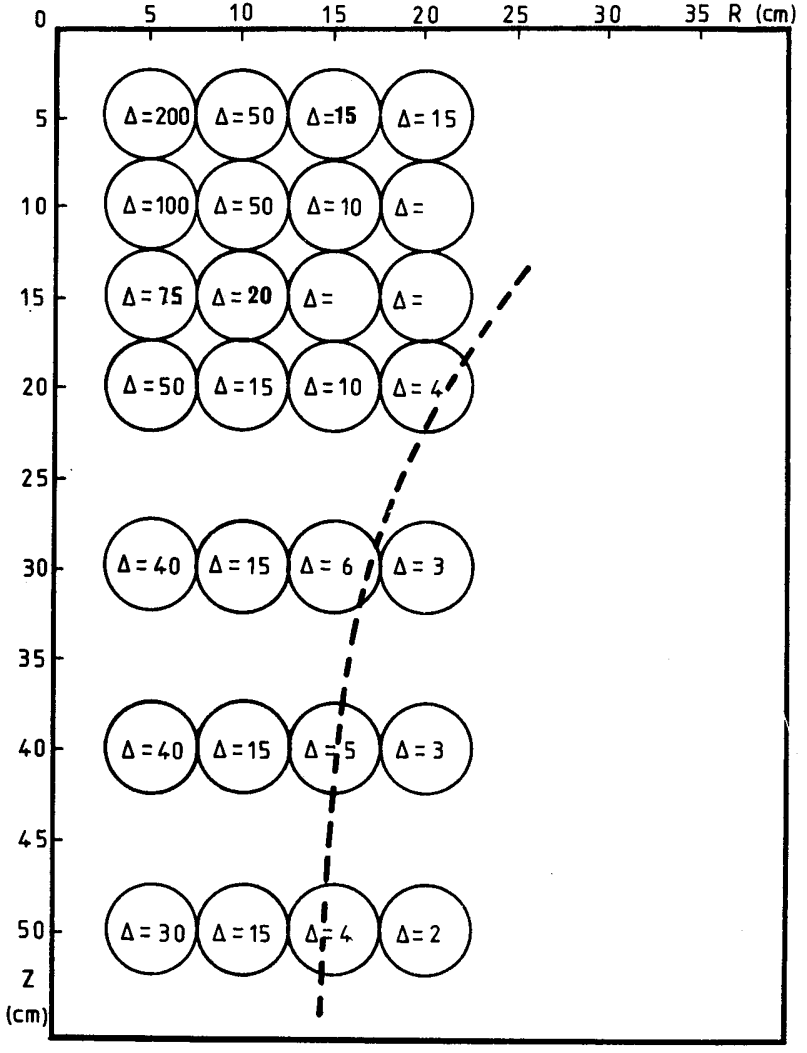

Fig. 2 Essai sur modèle : anomalies en \% créées par une cavité cylindrique de $5 \mathrm{~cm}$ de diamètre

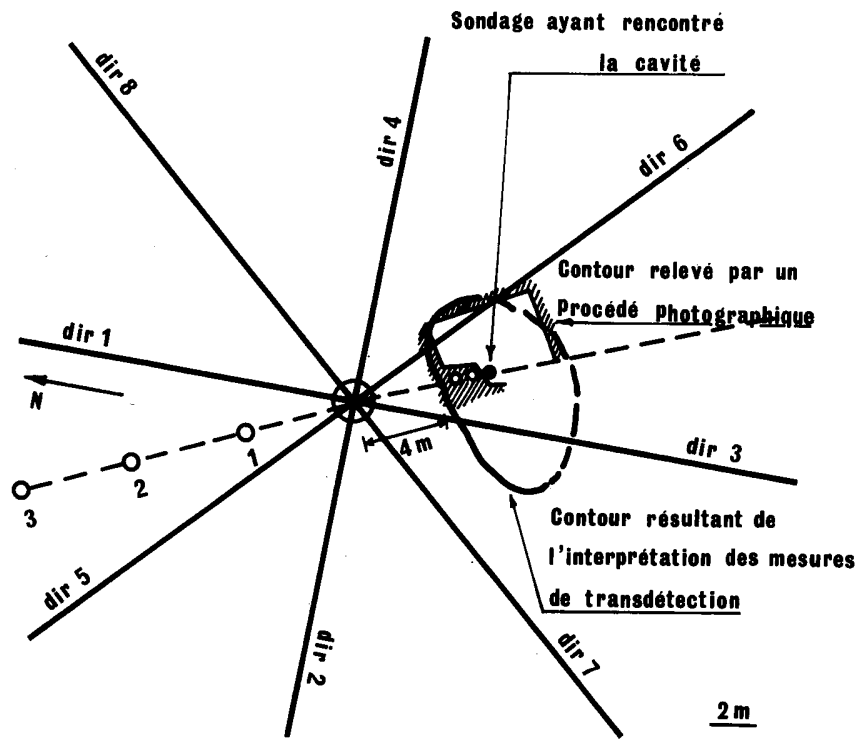

Fig. 3 Schéma d'implantation (Commune de Troux)

d'une hétérogénéité localisée revient donc à la recherche d'une anomalie dans la symétrie. La méthode que nous avons employée sur le terrain repose sur cette remarque.

\subsection{Les mesures à Troux}

Sur le site de Troux, la fig. 3 représente le schéma d'implantation des profils d'injection de courant. Nous avons effectué des mesures dans 8 directions, notées de 1 à 8 , sur lesquelles nous avons placé des prises de courant, la première à $5 \mathrm{~m}$ du puits, puis de $2 \mathrm{~m}$ en $2 \mathrm{~m}$, jusqu'à $36 \mathrm{~m}$. Le puits avait $22 \mathrm{~m}$ de profondeur.

Ne connaissant pas à priori ni la direction de la cavité, ni la résistivité moyenne des terrains, nous avons défini un indice d'anomalie pour deux directions opposées $i$ et $j$ par :

$$
\Delta=2 \frac{\rho_{\mathrm{i}}-\rho_{\mathrm{j}}}{\rho_{\mathrm{i}}+\rho_{\mathrm{j}}}
$$


La figure 4 résume les mesures faites pour une distance sondage-émetteur égale à 20 mètres. Une étude sur cuve analogique, en employant un modèle cylindrique plat surmonté d'un tronc en cône pour simuler une marnière en cours d'effondrement, a montré que dans ce cas le maximum de l'anomalie se trouvait au voisinage de la profondeur de la cavité. La cavité éventuelle se trouverait donc entre 13 et $15,5 \mathrm{~m}$ de profondeur.

- Sur la fig. 3 nous avons reporté l'interprétation faite des mesures. Nous avons voulu contrôler nos résultats par un forage : il a fallu 3 forages pour traverser une cavité (chute d'outil entre $13 \mathrm{~m}$ et $15 \mathrm{~m}$ de profondeur). Le premier forage s'est en effet trouvé dans la paroi, le deuxième dans un pilier, la distance entre les forages était de $2,5 \mathrm{~m}$.

Un appareil photographique descendu par le forage dans la cavité a permis d'avoir une image et de tracer approximativement les contours.

- Pour compléter cette série d'expériences et puisque nous disposions d'une cavité connue, nous avons voulu déterminer la limite d'investigation latérale de la méthode. Aussi, nous avons foré 3 nouveaux puits espacés de $5 \mathrm{~m}$ entre eux, en s'éloignant de l'aplomb de la cavité (fig. 3), donc à 12,17 et $22 \mathrm{~m}$ de l'aplomb du centre de la marnière. Ces puits sont numérotés $0,1,2$, et 3 . Le puits 0 est à $7 \mathrm{~m}$ de la cavité et a permis de la découvrir. A chaque fois nous avons refait les mesures sur le seul profil passant par la cavité.

Nous avons recommencé les mesures dans le puits 0 , nous avons obtenu (deux mois plus tard) les mêmes indices d'anomalie que lors de la première campagne.

Dans le puits 1, l'indice d'anomalie maximum était encore à $13 \mathrm{~m}$ de profondeur, de $60 \%$. Cependant, cet indice est artificiellement majoré par le fait que l'on avait injecté beaucoup de boue dans le puits 2 , que cette boue ne tenait pas et qu'elle devait former aux alentours du puits 2 une masse conductrice perturbatrice.

Dans le puits 2, nous n'avons pas pu faire de mesures. Dans le puits 3 , la grosse anomalie conductrice constituée par le puits 2 a masqué tous les résultats.

- Sur ce même site, nous avons utilisé plusieurs forages de contrôle d'une campagne gravimétrique pour y faire de la T.D.E. C'est ainsi que nous avons découvert une nouvelle cavité à $8 \mathrm{~m}$ de profondeur sur une anomalie gravimétrique positive. II a fallu 5 forages de contrôle pour obtenir les chutes d'outils attendues. Les vides n'ont que de $0,50 \mathrm{~m}$ à $1 \mathrm{~m}$ d'épaisseur. II s'agit en fait d'une marnière effondrée et les vides remontent vers la surface. Le déficit de masse est le même que pour une marnière saine et la résistivité locale du terrain en reste affectée, ce qui explique que la T.D.E. permette de le déceler.

Ceci montre que la T.D.E. permet de détecter aussi bien les marnières saines que celles qui sont en voie d'effondrement.

La technique d'interprétation permet actuellement de prévoir correctement la profondeur des vides, mais elle ne permet pas de connaître précisément leur distance à l'axe de forage.

\subsection{Conclusion}

La télédiagraphie électrique est une méthode efficace pour valoriser les sondages. Elle doit permettre de détecter une cavité dans un cylindre de rayon de l'ordre de deux fois la dimension de la cavité recherchée. Son champ d'application sera donc :

- contrôle d'une campagne de recherches de cavités à partir de la surface : par exemple, si un sondage implanté sur une anomalie gravimétrique ne trouve pas de cavités, cette méthode permettra de confirmer sa présence et de préciser sa position.

- étude des fondations d'un ouvrage ou d'un bâtiment : la mise en œuvre de cette œuvre sera faite dans le ou les sondages d'étude de la capacité portante du sol. Actuellement, nous disposons d'un matériel pour réaliser des mesures précises, il reste à perfectionner la mise en œuvre et l'interprétation pour diminuer les coâts d'étude.

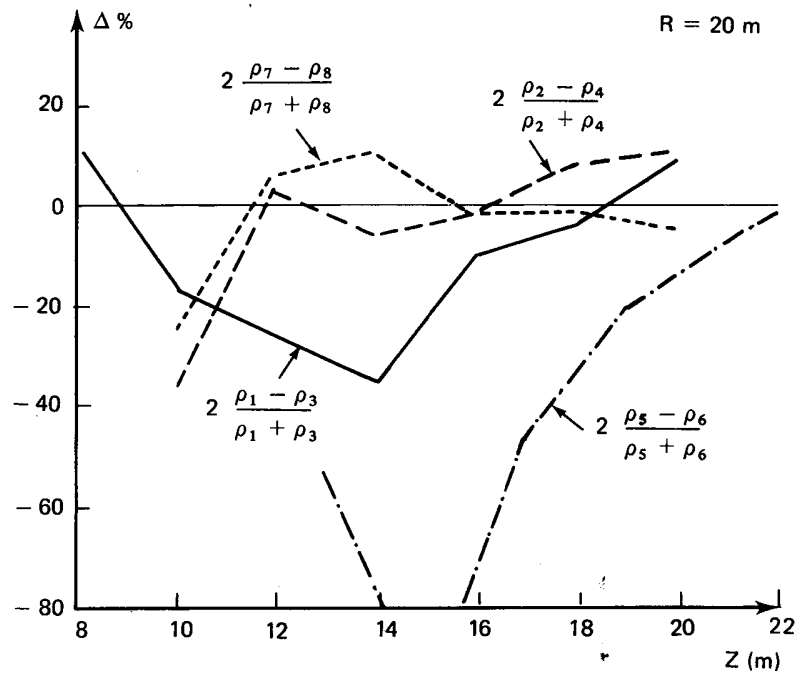

Fig. 4 Anomalies mesurées dans différentes directions (sondage 0)

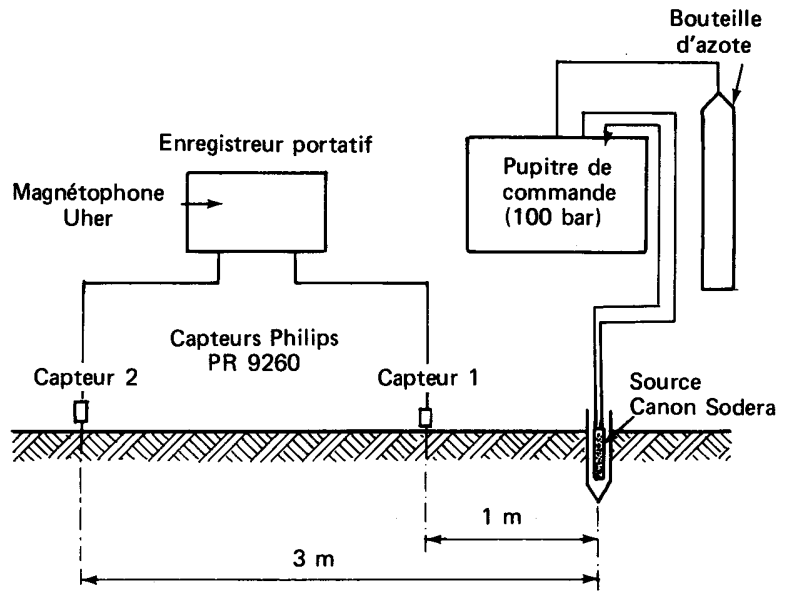

Fig. 5 Dispositif sismique utilisé sur le site de Troux (Bande passante de l'enregistrement : $50-1000 \mathrm{~Hz}$ )

\section{Le trainé sismique - réflexion}

\subsection{Principe}

En chaque nœud d'un réseau à maille carrée (d'environ $5 \mathrm{~m}$ ), on place successivement le dispositif de mesure sismique, comme suit : (fig. 5 ).

La source est un canon à air placé verticalement dans un trou plein d'eau. On place, alignés avec la source, deux géophones à $1 \mathrm{~m}$ et $3 \mathrm{~m}$ de celle-ci. Les géophones sont reliés à un magnétophone à 2 pistes qui enregistre les signaux. Soient $f_{1}(t)$ et $f_{2}(t)$ les signaux enregistrés respectivement au géophone 1 à $1 \mathrm{~m}$ et au géophone 2 à $3 \mathrm{~m}$. Examinons les deux cas :

a) Le terrain est homogène au voisinage du dispositif. b) II contient une cavité sous le dispositif.

Dans le cas a), les signaux $f_{1}(t)$ et $f_{2}(t)$ sont déphasés (les ondes de volume ou de surface arrivent en 2 avec un temps de retard par rapport à leur arrivée en 1) et on n'observe aucune corrélation entre $f_{1}(t)$ et $f_{2}(t)$. Leur produit $g(t)=f_{1}(t)$. $f_{2}(t)$ prend des valeurs faibles quelque soit $\mathrm{t}$.

Dans le cas b), une partie de l'énergie sismique est réfléchie sur le toit de la cavité qui se trouve loin des géophones vis-à-vis de la longueur du dispositif. Cette énergie réfléchie arrive donc en phase aux deux géophones, tandis que l'énergie transmise directement par la surface arrive encore déphasée. II y a donc une corrélation entre les deux signaux. Le produit $g(t)=f_{1}(t)$. $f_{2}(t)$ prend donc des valeurs fortes à partir de l'instant $T$ correspondant au trajet aller et retour de l'onde réfléchie. 
Ce calcul de $\mathrm{g}(\mathrm{t})$ est un critère de la présence d'une cavité. Remarquons que si la cavité était peu profonde, le déphasage subsisterait et que $g(t)$ prendrait des valeurs faibles. Par contre, $f_{1}(t)$ et $f_{2}(t)$ prendraient des valeurs fortes, car toute l'énergie sismique resterait confinée dans un volume faible. La cavité serait quand même détectée.

A partir de ces données, on peut tracer la carte d'anomalie sismique, qui fait apparaître les cavités. Ainsi, sur le site de Troux, la méthode met parfaitement en évidence deux cavités connues.

\subsection{Conclusion}

Cette méthode a permis de mettre en évidence des cavités. II reste cependant à mieux connaître les phénomènes mesurés pour connaître ses limites. Sa mise en œuvre est lourde; l'emploi du canon à eau nécessitant la réalisation de sondages peu profonds, mais nombreux (méthode de trainé). Son développement demande la mise au point d'une source légère.

\section{Magnétotellurique artificielle (M.T.A.)}

\subsection{Principe}

Brièvement cette méthode consiste à mesurer une résistivité apparente du sous-sol, en comparant les valeurs du champ électrique (qui est horizontal) et de la composante horizontale perpendiculaire du champ magnétique émis par un émetteur radio artificiel lointain sous une fréquence fixe (Guineau, Dupis, Carnez). L'appareil de mesure dont nous disposons est un prototype réalisé par le Centre de Recherches Géophysiques de Garchy qui permet de recevoir les émissions d'Allouïs $(163,84 \mathrm{kHz})$ et de Châteauroux $(15,07 \mathrm{kHz})$.

\subsection{Mesures}

Nous avons tracé une carte de résistivité apparente pour $164 \mathrm{kHz}$, les mesures pour $15 \mathrm{kHz}$ se sont révélées impossibles à réaliser par suite de mauvaises conditions de transmission de l'onde électromagnétique. Cette carte révèle une résistivité moyenne d'environ $10 \Omega$.m, ce qui est très faible et provient du fait que le limon de surface était gorgé d'eau. On a pu distinguer cependant une anomalie localisée au voisinage de l'aplomb de la cavité découverte plus tard par télédiagraphie électrique.

\subsection{Critique de l'emploi de cette méthode à Troux}

II s'avère qu'il s'agit sans doute d'une coïncidence. En effet, pour une résistivité de $10 \Omega . \mathrm{m}$, et une fréquence de l'onde de $164 \mathrm{kHz}$, la profondeur de pénétration n'est que de $3,90 \mathrm{~m}$, ce qui signifie qu'il ne faut pas espérer déceler une hétérogénéité localisée, dont le toit est à plus de $2 \mathrm{~m}$ de profondeur. II est possible que l'anomalie que l'on a mesurée résulte d'une conséquence superficielle de la présence de la cavité à $14 \mathrm{~m}$ de profondeur, mais alors elle est fugitive et dépend beaucoup des conditions d'humidité du terrain: d'ailleurs on ne l'a pas retrouvée lors d'une deuxième campagne de mesures. On n'a pas décelé non plus sur la carte d'anomalies la deuxième cavité effondrée découverte à $8 \mathrm{~m}$ de profondeur.

Enfin, si l'on avait pu faire les mesures à $15 \mathrm{kHz}$, on n'aurait pas non plus décelé de cavité, car la profondeur de pénétration de l'onde est de $13 \mathrm{~m}$ pour $10 \Omega \mathrm{m}$. II aurait donc fallu que le toit de la cavité soit à moins de $6,5 \mathrm{~m}$. En effet, si l'on considère un modèle de terrain constitué d'une couche de surface homogène conductrice de résistivité $\rho$ et d'épaisseur $h$ sur une couche de résistivité infinie, la résistivité apparente vaut $20 \%$ de plus que la résistivité du premier terrain, quand $h$ vaut la moitié de la profondeur de pénétration de l'onde (L. Cagniard); ceci pour une couche résistante d'extension latérale infinie. Pour une cavité, la résistivité apparente sera nécessairement plus faible et l'on ne peut pas espérer la déceler à une profondeur plus grande que la moitié de la profondeur de pénétration de l'onde.

\subsection{Conclusion}

En conséquence, il paraît souhaitable d'utiliser la MTA, par ailleurs très efficace, avec un émetteur portatif à environ $2 \mathrm{kHz}$, de manière à obtenir une profondeur de pénétration suffisante dans les terrains sédimentaires très conducteurs, tels que celui de Troux (la profondeur de pénétration de l'onde serait alors de $35 \mathrm{~m}$ pour une résistivité de $10 \Omega . \mathrm{m}$ ). II resterait encore à vérifier que les cavités ne sont pas trop petites pour être quand même détectées. Si la réponse était favorable, on disposerait alors d'une méthode de reconnaissance extensive très rapide et peu coûteuse. Cette méthode est par ailleurs très efficace pour les cavités superficielles, comme les catiches de la région lilloise (Carnez, 1976).

\section{Méthode électromagnétique surface-surface de détection par focalisation des courants à une profondeur déterminée}

\subsection{Principe}

La méthode est dérivée de la méthode aipôle-dipôle étudiée par $M$. le Professeur Gabillard. Dans cette méthode, on émet à la surface du sol un champ magnétique au moyen d'une boucle dans laquelle circule un courant à une fréquence variant de $5 \mathrm{kHz}$ à $95 \mathrm{kHz}$, deux autres boucles permettent de mesurer à une distance fixe du dipôle émetteur les composantes du champ radial $\mathrm{H}_{r}$ et vertical $\mathrm{H}_{\mathrm{z}}$, ainsi créé.

On compare ensuite le rapport $\mathrm{H}_{\mathrm{r}} / \mathrm{H}_{\mathrm{Z}}$ à celui qu'on obtiendrait en l'absence de cavités. On peut ainsi déplacer l'ensemble du dispositif sur le terrain à prospecter et classer les points de mesures grâce aux courbes de variation de $\mathrm{H}_{\mathrm{r}} / \mathrm{H}_{\mathrm{Z}}$, en fonction de la fréquence d'émission.

La figure 6 donne un exemple des courbes obtenues en présence et en l'absence de cavités avec cette méthode (les essais ont été effectués à Guyancourt, pour des cavités du même type que celles de Troux). On voit sur cette figure que la courbe 1 se trouve en dessous de la courbe 2 , ce qui laisserait supposer, d'après la théorie, que dans la zone de la courbe 1, la présence des cavités est plus probable que dans celle de la courbe 2 . Or, nous savons qu'il n'en est rien. Cette inversion provient du fait qu'au dessus des cavités existe une zone de remblais plus conductrice que le terrain correspondant dans les zones dépourvues de cavités.

Pour améliorer la méthode, I'Université des Sciences et Techniques de Lille a essayé de focaliser les courants induits par l'émetteur à la profondeur présumée des cavités, ceci afin de privilégier l'effet des couches contenant les anomalies aux dépens des couches superficielles qui peuvent, comme on l'a vu, compenser les effets des cavités. Pour cela, on utilise une boucle émettrice secondaire plus petite que la boucle principale et située à une distance déterminée de celle-ci. Dans cette boucle, on émet un courant, de même fréquence, que celui qui circule dans la boucle principale mais déphasé de $\pi$. On règle ensuite son intensité, de sorte que le champ électrique induit au voisinage de l'émetteur soit minimum à $100 \mathrm{kHz}$, donc à une fréquence où il intéresse essentiellement les couches de surface. On fait ensuite un balayage en fréquence à l'émission, en gardant asservie l'émission de la boucle secondaire. La théorie montre que le courant induit dans le sol est focalisé à une profondeur que l'on peut calculer en fonction des fréquences et de la géométrie du dispositif.

La figure 7 montre les courbes obtenues à Guyancourt aux mêmes points que précédemment. On voit que pour les basses fréquences, on obtient des résultats comparables à ceux de la méthode dipôle-dipôle, par contre autour de $50 \mathrm{kHz}$ la courbe 1 (sans carrière) indique des valeurs plus fortes que la courbe 2 (avec carrières), l'effet de masque des matériaux de surface est complètement supprimé et la différence des valeurs de $\mathrm{H}_{\mathrm{r}} / \mathrm{H}_{\mathrm{Z}}$ considérablement augmentée.

Au dessus des carrières, on a fait 3 mesures indépendantes 


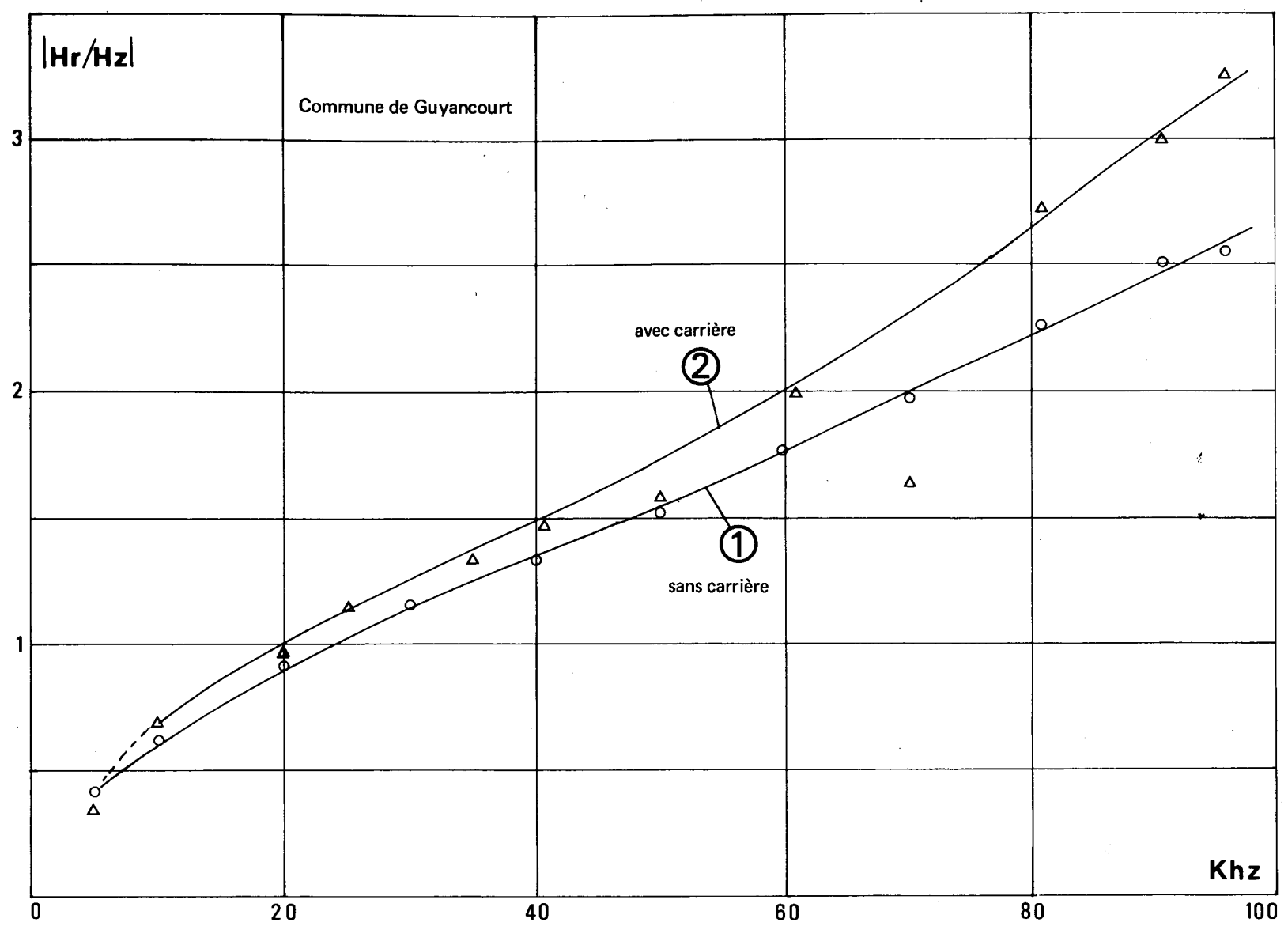

Fig. 6 Recherche de cavités à Guyancourt : mesures sans focalisation de courant

Fig. 7 Recherche de cavités à Guyancourt : mesures avec focalisation de courant

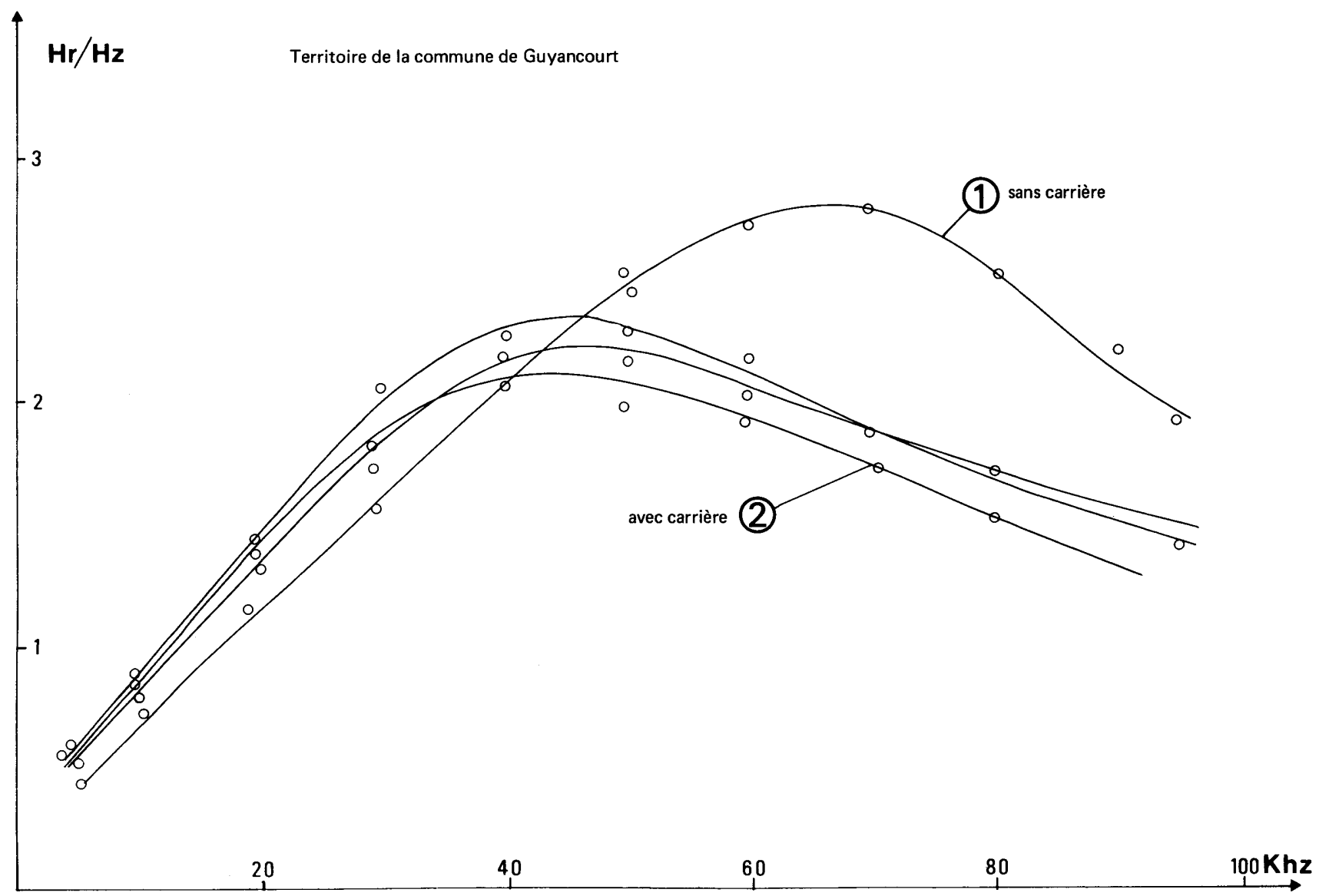


pour avoir une idée de l'erreur : elle reste très inférieure à la valeur de l'anomalie.

\subsection{Conclusion}

Cette méthode semble séduisante : les expériences effectuées confirment la théorie, c'est-à-dire que le pouvoir de séparation de cette méthode doit être quatre fois supérieur à celui de la méthode dipôle-dipôle; son inconvénient majeur, actuellement, est le temps de mise en œuvre. Si l'on parvenait à rendre la mise en phase plus rapide, on aurait sans doute affaire à une méthode puissante.

\section{Conclusion}

Sur un site connu, nous avons pu tester différentes méthodes de prospection des cavités souterraines. Celles que nous recherchions étaient petites et situées à profondeur moyenne. II n'est pas possible d'extrapoler les conclusions de ce site à tous les autres sites. Des méthodes comme la magnétotellurique artificielle, la gravimétrie n'ont pas donné satisfaction, alors que l'on sait qu'elles conviennent parfaitement dans d'autres conditions (par exemple, la détection de catiches dans la région lilloise). II importe donc de choisir sur chaque site la méthode qui est la plus appropriée. Si l'on connaît assez bien les limites de certaines méthodes (gravimétrie, M.T.A.), il n'en est malheureusement pas de même pour les autres. Les mesures réalisées sur le site de Troux fournissent donc des indications intéressantes. Toutes les méthodes essayées, autres que celles déjà citées ont permis de déceler plus ou moins facilement des cavités :

- Le trainé sismique réflexion a donné de bons résultats, mais il convient d'améliorer sa mise en œuvre, principalement au niveau de la source (canon à eau). Les essais sur maquette n'ont pas permis d'apprécier les limites de cette méthode et il serait nécessaire de poursuivre l'étude théorique.

- La méthode par focalisation des courants électriques a été testée et on a ainsi vérifié la théorie, c'est-à-dire que le pouvoir détecteur de cette méthode devait être quatre fois supérieur à celui de la méthode classique dipôle-dipôle. Elle est ainsi supérieure à la gravimétrie. Cependant, il convient d'améliorer sa mise en œuvre.

- La télédiagraphie électrique permet de valoriser considérablement les sondages. Les mesures effectuées ont permis de vérifier l'exactitude des prévisions faites sur maquette. Pour l'avenir, il conviendrait d'améliorer l'interprétation de la mesure et de diminuer le nombre de mesures à faire sur le terrain, pour que son coût ne soit pas trop élevé.

Nous remercions la D.G.R.S.T., qui par son aide, a permis la réalisation de cette recherche.

\section{Références Bibliographiques}

BAVANDI R. - Étude théorique et expérimentale de méthodes électromagnétiques de détection des cavités souterraines permettant de valoriser les informations obtenues par des sondages mécaniques. Thèse de $3^{\circ}$ cycle. Lille 1976.

CAGNIARD L. - Principe de lo méthode magnétotellurique, nouvelle méthode de prospection géophysique. Ann. Geophys., 9, pp. 95-125, 1953.

CARNEZ J.-L., DUPIS A. - Application d'une méthode magnétotellurique a la reconnaissance des sites. Bull. Liaison LPC, mai-juin 1976, $n^{\circ} 84$. Comité Français de Géologie de l'Ingénieur - Séance du 10 février 1977. "Cavités " Bull. Liaison LPC nov.-déc. 1977, nº 92.

DUBUS J.-P., GABILLARD R., BAVANDI R., RAT $M$., LAGABRIELLE R. - Détection des cavités souterraines par une méthode électromagnétique entre surface et forage. Revue de l'Association Internationale de Géologie de l'Ingénieur, juin $1977, \mathrm{n}^{\circ} 15$.

DUBUS J.-P., BAVANDI R., MANGEZ P. - Rapport sur une campagne de détections de cavités souterraines pratiquées dans le département des Yvelines. USTL/LCPC. Avril 1976.

GABILLARD R. - Propagation des ondes électromagnétiques dans les milieux conducteurs. Institut de radioélectricité, Lille.

GABILLARD R., LOUAGE F., FONTAINE J., DUBUS J.-P.Théorie matricielle de la propagation dans un terrain stratifié d'une onde électromagnétique émise par une antenne verticale souterraine. Symposium sur les ondes électromagnétiques, Stresa, juin 1963.

GUINEAU B. - Application de la méthode magnétotellurique de prospection géophysique à l'étude des couches très superficielles du sous-sol. These $3^{\circ}$ cycle - Paris VI, 1973.

GRISONI. - Synthèse régionale du calcaire de Beauce. Rapport interne LCPC.

LOUAGE F. - Faisabilité d'un procédé de détection de cavités souterraines LCPC/USTL 1973.

PERAGALLO J. - Méthodes sismiques de subsurface : contribution à la détection des cavités souterraines. Thèse de $3^{\circ}$ cycle - Bordeaux I, 1976. 